\title{
Plataforma de Brazos Robóticos de Tecnología Abierta para realizar Experiencias de Laboratorio de Control de Posición, Planificación de Trayectoria y Control de Visión Artificial
}

\author{
Robotic Arms Plataform Technology open to Perform Laboratory \\ Experiences in Control of Position Career Planning and Control of \\ Artificial Vision
}

\author{
Mario Borja Borja ${ }^{1 *}$, Javier Rojas Tintaya ${ }^{1}$, Rodney Rodas Regalado ${ }^{1}$, Carlos Díaz Ramirez ${ }^{1}$, Cesar Paz \\ Chavez $^{1}$ \\ ${ }^{1}$ Facultad de Ingeniería Mecánica, Universidad Nacional de Ingeniería, Lima, Perú
}

\begin{abstract}
RESUMEN
El principal problema en el desarrollo de carreras de especialización en robótica en nuestro país es que no existen laboratorios con brazos robóticos que permitan hacer pruebas de control de posicionamiento, planificación de trayectoria y control con visión artificial y generalmente en entornos académicos se hace experimentos utilizando software de simulación.

En el presente trabajo se propone una alternativa para mejorar el aprendizaje en el estudio de control de brazos robóticos utilizando brazos robóticos reales con una plataforma de tecnología abierta de software y hardware que permita a los estudiantes modificar los programas para implementar algoritmos de control de posición de articulaciones, planificación de trayectoria y control con visión artificial.

La propuesta es desarrollar la tecnología total del sistema de control del brazos robóticos en la base al análisis de los requerimientos de electrónica y sistema de computo realizar la selección de electrónica de potencia, computadoras industriales basadas en Controlador Digital de señales (DSC), una computadora personal para interface de usuario.

Desarrollar el software base para la computadora industrial y el software base para la computadora personal que permita cargar programas en línea a través del puerto serial desde una computadora personal y además una interface de usuario en la computadora que puede ser modificada de acuerdo a la necesidad y utilizar como sistema de control de nivel superior que realiza los cálculos de planificación de trayectoria, problema inverso y otros sistemas de alto nivel de control con visión artificial.

Como resultado se obtuvo la plataforma de tecnología abierta que incluye hardware y software para brazos robóticos de hasta seis grados de libertad con motores de corriente continua en las articulaciones de hasta 100 vatios, encoders y sensores de fin de carrera.

La plataforma se probó con la mecánica del brazo robótico serial Mitsubishi RV-M1 que cumple con los parámetros electrónicos y constructivos requeridos por el prototipo.

Palabras claves: brazos robóticos; control de posición; plataforma abierta
\end{abstract}

\begin{abstract}
The main problem in the development of specialization courses in robotics in our country is that there are no laboratories with robotic arms that allow testing positioning control, path planning and control with artificial vision and generally in academic settings is done experiments using software simulation.

In this paper an alternative to enhance learning in the study control robotic arms using real robotic arms with an open technology platform software and hardware that allows students to modify programs to implement control algorithms position it is proposed joints, path planning and control with artificial vision.

The proposal is to develop the full technology control system of the robotic arms on the basis of the analysis of the requirements of electronic and computer system the selection of power electronics, industrial computers based on digital signal controller (DSC), a computer personnel to user interface.

Develop the core software for industrial computer and basic software for the PC that allows load programs online through the serial port from a personal computer and also a user interface on the computer that can be modified according to the need and used as control system upper level that performs calculations path planning, inverse problem and other high-level systems with artificial vision control.

As a result the open technology platform that includes hardware and software for robotic arms up to six degrees of freedom with DC motors in the joints of up to 100 watts, encoders and sensors to run was obtained. The platform was tested with the mechanics of serial robotic arm Mitsubishi RV-M1 compliant electronic and construction parameters required by the prototype.
\end{abstract}

Keywords: robotic arms; position control; open platform

La Revista Científica TECNIA protege los derechos de autor bajo la Licencia 4.0 de Creative Commons: Attribution 4.0 International (CC BY 4.0). 


\section{INTRODUCCIÓN}

Los brazos robóticos de diversas con Figura ciones son muy aplicados en la industria en la asistencia en diversos procesos de fabricación.

En nuestro país se está iniciando la utilización de los mismos en la industria nacional para mejorar la calidad y competitividad de nuestros productos. Estos brazos robóticos son importados de empresas de países desarrollados como EEUU, países europeos y asiáticos, los cuales entre sus características principales son de alto costo por tratarse tecnología de última generación y son sistemas totalmente cerrados que solo es posible operar.

Por otro lado en las especialidades de electrónica y mecatrónica de las universidades en nuestro país se dictan cursos de robótica, sin embargo generalmente para hacer pruebas de laboratorio se realiza simulaciones debido a que en los laboratorios académicos no existes brazos robóticos con sistemas de tecnología abierta que permita a los estudiantes realizar pruebas de los diversos temas como: Planificación de trayectoria [1], problema cinemático inverso [1][2][3][5][6][7], control [1][2][3][6][6] [7] y control con visión artificial, que ayudaría en el entendimiento, entrenamiento y experiencia para diseñar, construir y controlar brazos robóticos.

Uno de los aspectos importantes en el diseño y construcción de brazos robóticos es el sistema de control que incluye planificación de trayectoria, problema cinemático inverso y control de posición de cada articulación, el cual es un software que corre sobre un procesador industrial (Microcontroladores, procesadores digitales de señales o procesadores digitales de control) combinado con una interface de usuario en una computadora personal.

Generalmente en los procesadores industriales se ejecuta la parte de software de control de posición de las articulaciones, este recibe la referencia de posición y mediante algoritmos de control, posiciona cada articulación haciendo que el brazo robótico alcance el punto deseado en el espacio.

Para esto los procesadores industriales actúan directamente sobre la electrónica de potencia que alimentan los actuadores de las articulaciones.

Correspondencia:

*E-mail:mborja33@hotmail.com
La interface de usuario de la computadora personal permite que el usuario ingrese la posición o trayectoria deseada y realiza el cálculo del problema inverso para obtener la posición que debe moverse cada articulación para que el brazo robótico alcance

la posición deseada, esta posición deseada es la referencia para los controladores de cada articulación.

Para que los estudiantes tengan la oportunidad de realizar pruebas de laboratorio y entrenarse en todas las fases de control del brazo robótico se requiere una tecnología abierta de software que tenga la estructura básica del sistema y tengan la posibilidad de modificar e implementar nuevos algoritmos de planificación de trayectoria, problema inverso y controladores de posición de las articulaciones del brazo robótico utilizando visión artificial.

\section{FUNDAMENTOS TEÓRICOS}

Para el diseño de prototipo se realizó un análisis de todos principios teóricos que sustentan el funcionamiento de cada componente del brazo robótico con el objetivo de determinar las características de hardware y software que debe incluir el sistema.

\section{A. Cinemática}

La cinemática permite representar matemáticamente los movimientos de los componentes móviles del brazo robótico.

Si partiendo de las coordenadas generalizadas se obtiene loas coordenadas cartesianas del efector final, se conoce como el problema cinemático directo y viceversa si utilizando las coordenadas cartesianas de ubicación de efector final se calcula las coordenadas generalizadas se conoce como problema cinemático inverso [1].

\section{B. Dinámica}

Ladinámicadel brazo robóticoes la representación matemática de comportamiento de los torque y fuerzas que influyen en el movimiento de los diferentes componentes mecánicos [1].

\section{Controlador digital de señales}

El controlador digital de señales (DSC) es una computadora industrial que tiene temporizadores, PWMs, lector de encoders, etc, diseñado 
específicamente para implementar sistemas de control de motores y procesos industriales [9] [10] [11] [12] [13].

\section{Visión artificial}

La visión artificial se desarrolla en base al análisis de imágenes capturadas por medio de cámaras, en donde cada pixel es analizado para obtener características relevantes que nos ayudan a resolver el problema propuesto.

\section{Prototipo}

La construcción del prototipo se realizó con el brazo robótico serial presentado en la Figura 1 y considerando que el control de posición de articulaciones se realiza en el Controlador digital de señales (DSC) por software y controla los amplificadores de potencia realizados como puentes $\mathrm{H}$, la cinemática inversa y la planificación de trayectoria con o sin visión artificial se implementa en la computadora personal (PC), entonces la electrónica y sistema de cómputo del sistema de control es universal para cualquier conFigura ción geométrica con cualquier número de articulación siempre y cuando la potencia de los motores sea menor o igual a la potencia de los puentes $\mathrm{H}$ utilizados.

Para realizar pruebas de laboratorio de control de posición de las articulaciones, cinemática inversa, planificación de trayectoria con visión artificial el prototipo tiene las siguientes características:

- Para implementar algoritmos de planificación de trayectoria con visión artificial el sistema tiene el software para capturar las fotos desde dos cámaras, conservarlo en archivos y el pre procesamiento de imágenes.

- Elsistematieneelsoftwarequeparalaplanificación de trayectoria y problema cinemático inverso para obtener las coordenadas generalizadas del robot que serán enviadas al DSC que realiza el control de articulaciones.

- La trasmisión de las coordenadas generalizaras desde la computadora personal al DSC debe estar debidamente funcional de tal forma que si se escribe los datos en un buffer este sea inmediatamente trasmitido al DSC para que posicione el brazo robótico en la posición deseada.

- El software de control de posición de articulaciones tienen una estructura que solo es necesario cambiar el programa de la ley de control de acuerdo a lo

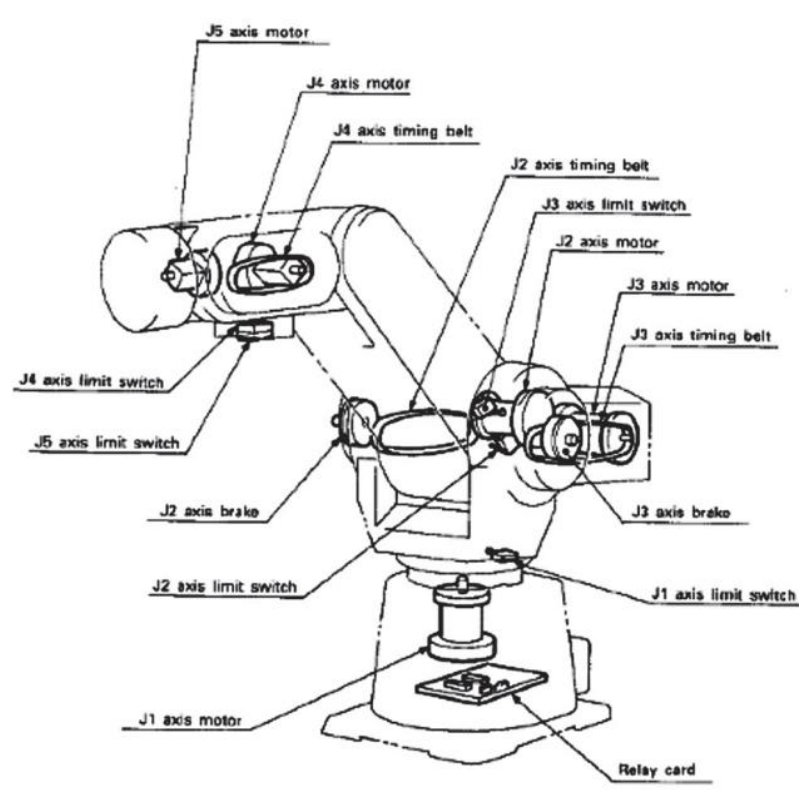

Figura 1. Diagrama del brazo robotico articulado de cinco grados de libertad.

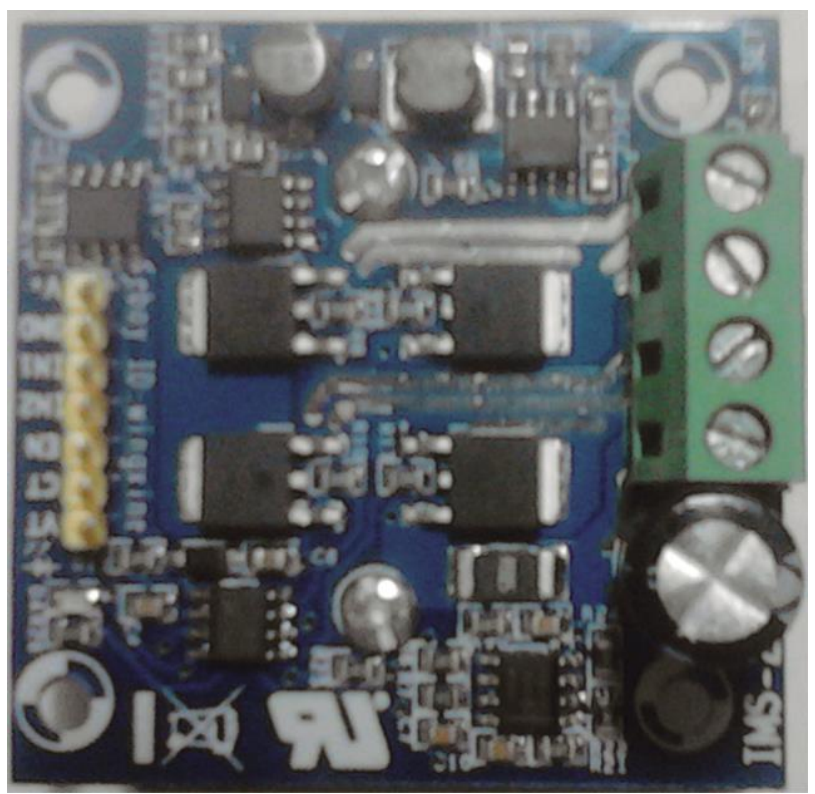

Figura 2. Puente $\mathrm{H}$ de 120 vatios.

que se desee experimentar y el sistema tome las referencias de posición enviadas desde la PC, mide las posiciones actuales, calcula el resultado de control y envía en forma de PWM a través de los puentes $\mathrm{H}$ a los motores para alcanzar la posición deseada.

El prototipo construido permite elcontroldecualquier brazo robótico de hasta seis grados de libertad que las articulaciones sea actuadas por motores de corriente continua de hasta 100 vatios, y tengan encoders 


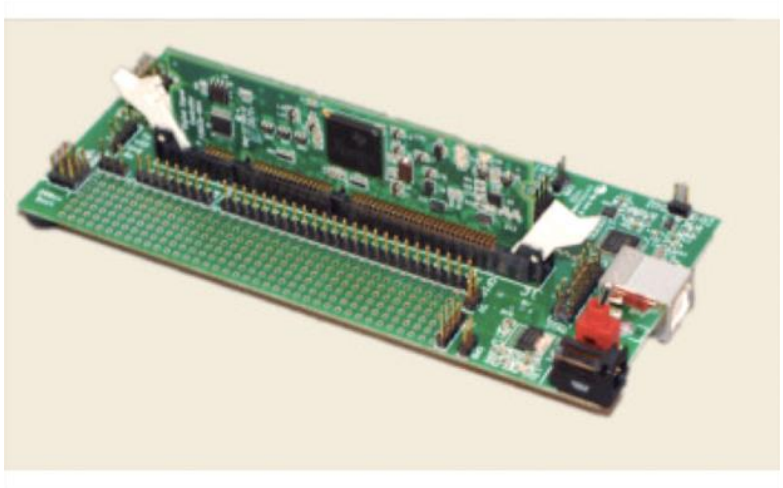

Figura 3. Procesador digital de señaales (DSC).

incrementales en las articulaciones y sensores de fin de carrera.

\section{E. Sistema electrónico}

El sistema electrónico del prototipo controla la posición del motor de corriente continua por voltaje en el devanado de la armadura para esto

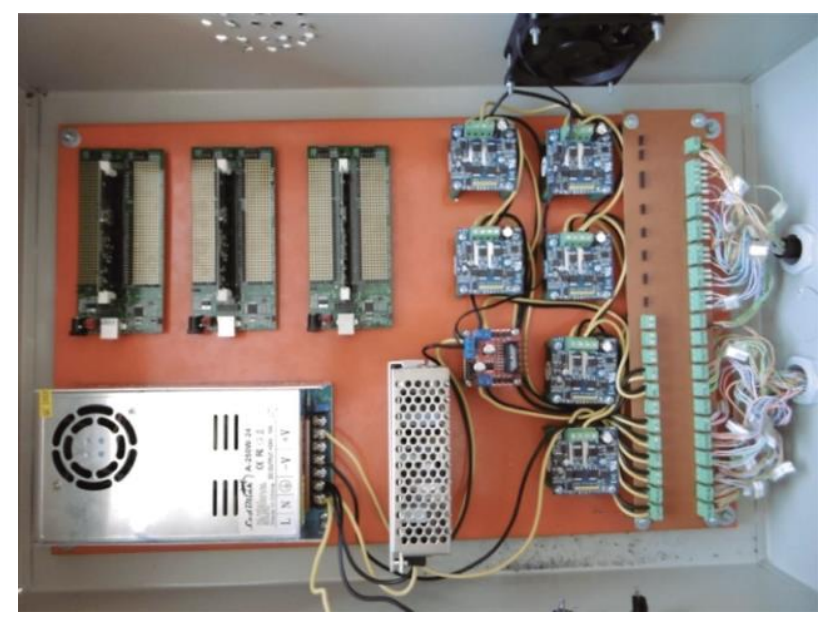

Figura 4. Caja de control.

usamos un puente $\mathrm{H}$ con el que se muestra en la Figura 2, haciendo un total de seis puentes $\mathrm{H}$, uno por cada motor de cada articulación como se muestra en la caja de control de la Figura 4.

Para realizar el control de posición de articulaciones utilizando diversas estrategias de control se utiliza un DSC que se muestra en la Figura 3, que mide la posición de la articulación desde las dos señales del encoder, recibe la referencia desde la computadora personal como resultado de la solución del problema inverso, realiza el cálculo del controlador y entrega la señal de control mediante PWM hacia el puente H.

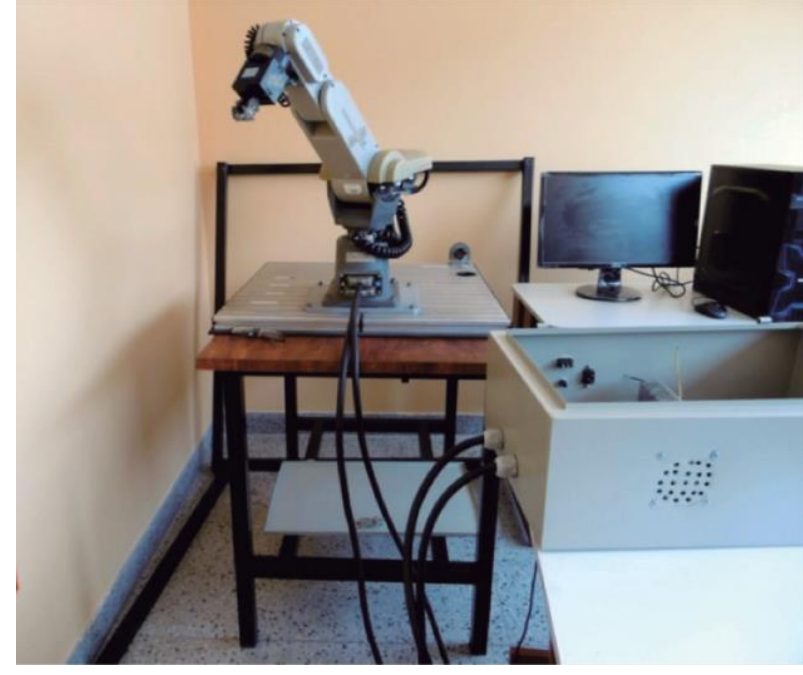

Figura 5. Prototipo de sistema de control.

El sistema de control completo como se ve en la Figura 5 consta de una computadora personal y la caja de control que se conecta al Brazo robótico.

Para realizar las pruebas se utilizó la mecánica del brazo robótico Mitsubishi RV-M1 de cinco grados de libertad muy común en los laboratorios académicos para experimentar planificación de trayectoria mediante la programación en el lenguaje del robot, con los motores originales, sensores de fin de carrera, frenos para la segunda y tercera articulación y la tarjeta de adaptación de señales que se conecta directo a la caja de control.

Con el sistema de control desarrollado se reemplazó toda la electrónica y software original del brazo robótico.

\section{F. Software del sistema de control}

El software del sistema de control del brazo robótico se organiza como se muestra en la Figura 6, en donde podemos ver que en el nivel superior esta la planificación de trayectoria y esta puede ser realizada usando un programa de robot programado en el lenguaje del robot o mediante visión artificial, la planificación de trayectoria da una secuencia de puntos en coordenadas cartesianas que aplicando el problema cinemático inverso obtenemos las coordenadas generalizadas delrobotyasean ángulos de giro odesplazamientos que hacen que el brazo robótico alcance el punto deseado.

Las coordenadas generalizadas son enviadas al sistema de control de articulaciones y son usadas 


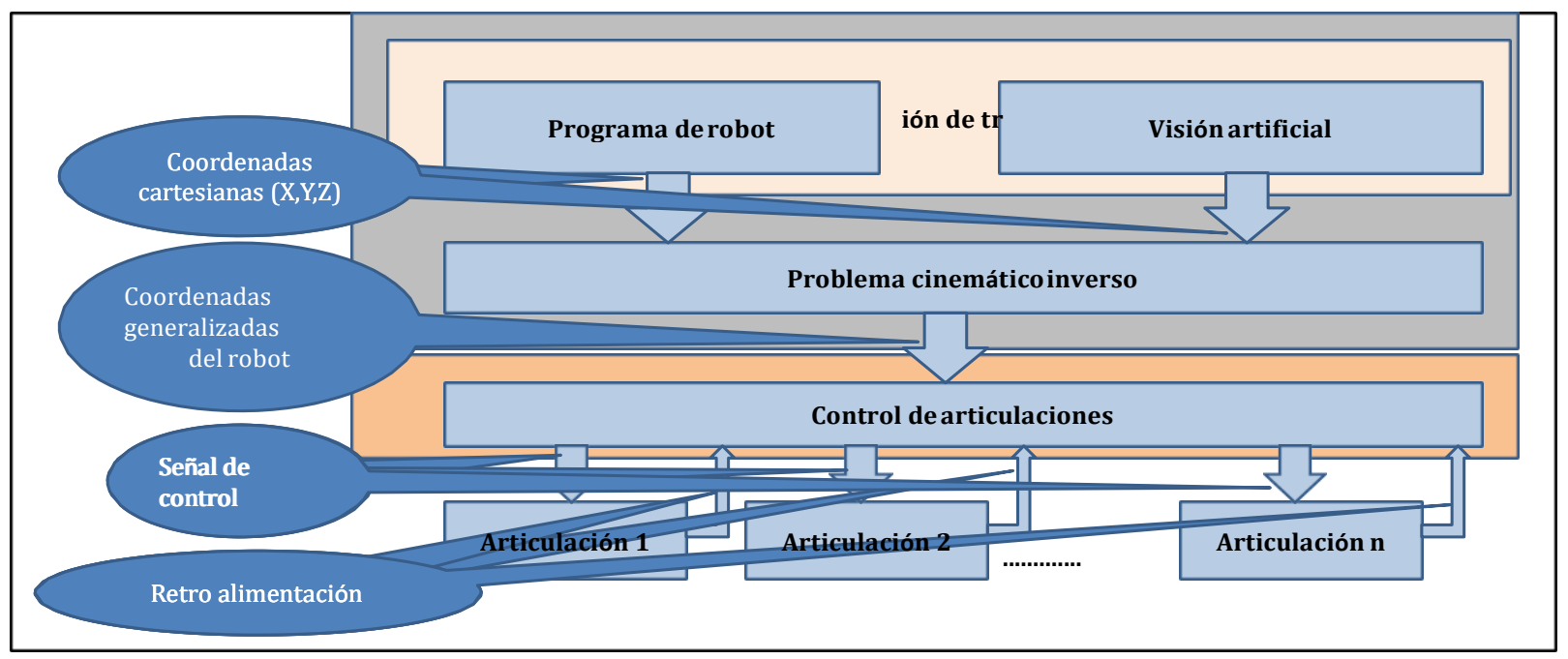

Figura 6. Prototipo de sistema de control.

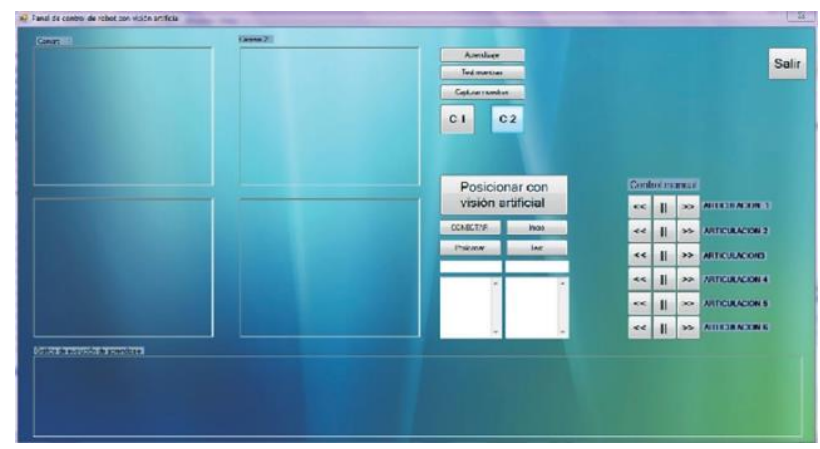

Figura 7. Panel de control del brazo robótico.

como referencia para que las articulaciones alcancen dicha posición.

Para crear el sistema que permita realizar experiencias en planificación de trayectoria, problema cinemático inverso y control de articulaciones se desarrolló como se muestra en la Figura un software base para computadora personal y para el DSC que incluye la programación general permitiendo que para probar un nuevo

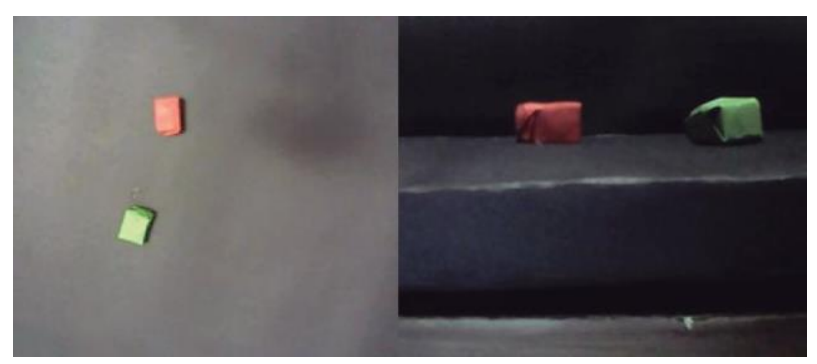

Figura 8. Muestras de captura de imágenes de vista superior y lateral con las cámaras y el panel de control. algoritmo solo sea necesario hacer el programa del algoritmo y ubicarlo en el lugar predefinido del programa correspondiente.

El panel de control del brazo robótico que se muestra en la Figura 7 es la interface del usuario con el robot, que permite realizar pruebas manuales de las articulaciones, inicializar el sistema, pruebas de posicionamiento, pruebas demovimiento.

En la Figura 8 se muestra un ejemplo de captura de imágenes mediante las dos cámaras instaladas en el prototipo de vista superior y vista lateral mediante el panel de control.

\section{RESULTADOS}

Se construyó la caja de control que s muestra en la Figura 4 que contiene los puentes $\mathrm{H}$ para ampliar la potencia de la señal de control, las fuentes de alimentación para los motores y frenos y los controladores digitales de señales para controlar las articulaciones del robot.

Se tiene un prototipo que incluye computadora personal, caja de control y robot interconectados como se muestra en la Figura 5.

El software para la computadora personal es el panel de control del brazo robótico como se muestra en la Figura 6 y con el que se puede hacer pruebas manuales de funcionamiento de las articulaciones, captura fotos con la dos cámaras como se presenta en la Figura 8 y envía los datos de coordenadas generalizadas desde la computadora personal hasta el DSC. 
El software de control de posición de articulaciones del DSC recibe las coordenadas generalizadas desde la computadora personal, mide la posición de cada articulación y genera el PWM de acuerdo a los resultados del algoritmo de control.

- La calidad física del prototipo obtenido considerando sus componentes electrónicos y ensamblado presenta una confiabilidad para usar sin inconvenientes en investigación de profesores, alumnos e interesados engeneral.

- El hardware y software desarrollado ofrece lo que se requiere estrictamente para realizar experimentos de investigación en planificación de trayectoria con visión artificial, problema cinemático inverso y control de articulaciones con mucha facilidad ya que el software base desarrollado para la computadora personal captura imágenes desde las cámaras y envía las coordenadas generalizadas al controlados digital de señales, el cual a su vez mide la posición real, calcula el controlador y envía los resultados por PWM evitando que el investigador desgaste recursos en actividades de desarrollo no relevantes para su investigación, permitiéndole solo dedicarse a los algoritmos de su interés.

- El software fue desarrollado en lenguajes de programación de uso común como son el visual C\# y el C++ evitando el uso de costosas licencias de software.

- El soporte técnico para el prototipo está garantizado ya que fue desarrollado por nuestro equipo de investigación.

- El prototipo es fácilmente replicable ya que durante el desarrollo se definió cada proceso para construir el prototipo.

- El sistema de control desarrollado puede ser usado en robots de cualquier conFigura ción hasta seis articulaciones que tengan motores de corriente continua de hasta 100 vatios sin realizar ningún cambio de hardware ysoftware.

- Si se necesita aplicar en un robot de mayor potencia entonces solo es necesario cambiar los puentes $\mathrm{H}$ y fuentes de alimentación de acuerdo a la potencia de sus actuadores.

\section{REFERENCIAS}

[1] Barrientos A y Balaguer C 1997 Fundamentos de Robótica Mc Graw Hill:España

[2] Borja M Sistema de Control de Brazo Robótico para experimentar la cinemática inversa y planificación de trayectoria en entornos académicos INTERCON 2014

[3] Cabana V E, Cuellar L N, Montes P M, Molero F R, Separovich M D and Borja B M 2008 Induction Motor Sliding Vector Control Using a DSP TMS320LF2407 Proceedings of MUSME 2008, the International Symposium on Multibody Systems and Mechatronics San Juan Argentina 12

[4] Dorsey J 2005 Sistemas de Control Continuos y Discretos: Modelado, Identificación, Implementación Mc GrawHill

[5] Cuellar N, Sotelo V y Borja M 2008 Diseño de un Controlador Nolinealde Posiciónpara un Servomotor Brushless ANDESCON 2008 Cusco Perú pp 1-7

[6] Cuellar Loyola N, Molero R, Borja M, Sotelo V 2008 Control Robusto de Trayectoria para un Manipulador Robótico usando Motores Brushless INTERCON2008

[7] Cuellar N, Borja M, Sotelo V 2009 Diseño de un control adaptativo robusto para el seguimiento de trayectorias de un robot industrial de 4 grados de libertad XVIII CONIMERA

[8] Lung-Wen T 1999 Robot analysis: The mechanical of serial and parallel manipulators

[9] Texas Instruments 2007 TMS320x2833x Analogto-Digital Converter (ADC) Module. SPRU812A (2007, October)

[10] Texas Instruments 2007 TMS320F28335, TMS320F28334, TMS320F28332, TMS320F28235, TMS320F28234, TMS320F28232, Digital Signal Controllers (DSCs) Literature Number: SPRS439H(2010, March)

[11] Texas Instruments. TMS320x2833x, 2823x System Control and Interrupts. SPRUFB0DSeptember 2007-Revised March 2010.

[12] Texas Instruments. TMS320x2833x, 2823x Enhanced Pulse Width Modulator (ePWM) Module. SPRUG04A (2009, July)

[13] Texas Instruments TMS320x2833x, 2823x Enhanced Quadrature Encoder Pulse (eQEP) Module. SPRUG05A (2008, December) 